\title{
OSCULATING QUADRICS OF RULED SURFACES IN RECIPROCAL RECTILINEAR CONGRUENCES
}

\author{
M. L. MACQUEEN
}

1. Introduction. Let $x$ be a general point of an analytic non-ruled surface $S$ referred to its asymptotic net in ordinary projective space. By a line $l_{1}$ at the point $x$ we mean any line through the point $x$ and not lying in the tangent plane of the surface at the point $x$. Dually, a line $l_{2}$ is any line in the tangent plane of the surface at the point $x$ but not passing through the point $x$. The lines $l_{1}, l_{2}$ are called reciprocal lines if they are reciprocal polar lines with respect to the quadric of Lie at the point $x$. In this case, when the point $x$ varies over the surface $S$, the lines $l_{1}, l_{2}$ generate two rectilinear congruences $\Gamma_{1}, \Gamma_{2}$ which are said to be reciprocal with respect to the surface. If, however, the point $x$ moves along the $u$-curve, the locus of the line $l_{1}$ is a ruled surface $R_{1}^{(u)}$ of the congruence $\Gamma_{1}$. The osculating quadric along a generator $l_{1}$ of the ruled surface $R_{1}^{(u)}$ is the limit of the quadric determined by the line $l_{1}$ through the point $x$ and the lines $l_{1}$ through two neighboring points $P_{1}, P_{2}$ on the $u$-curve as each of these points independently approaches the point $x$ along the $u$-curve. The quadric thus defined will be denoted by $Q_{1}^{(u)}$. A second quadric $Q_{1}^{(v)}$ is determined by three consecutive lines $l_{1}$ at points of the $v$-curve through the point $x$. Moreover, there are two quadrics, denoted by $Q_{2}^{(u)}$ and $Q_{2}^{(v)}$, which are associated with two ruled surfaces of the reciprocal congruence $\Gamma_{2}$ and which can be defined similarly. This note will study the projective differential geometry of the quadrics thus defined.

2. Analytic basis. Let the surface $S$ under consideration be an analytic non-ruled surface whose parametric vector equation, referred to asymptotic parameters $u, v$, is

$$
x=x(u, v) .
$$

The four coordinates $x$ of a variable point $x$ on the surface satisfy two partial differential equations which can be reduced, by a suitably chosen transformation of proportionality factor, to Fubini's canonical form

$$
x_{u u}=p x+\theta_{u} x_{u}+\beta x_{v}, \quad x_{v v}=q x+\gamma x_{u}+\theta_{v} x_{v}, \quad \theta=\log \beta \gamma,
$$

in which the subscripts indicate partial differentiation. The coefficients of these equations are functions of $u, v$ and satisfy three integrability conditions which need not be written here. 
Two lines $l_{1}, l_{2}$ are reciprocal lines if the line $l_{1}$ joins the point $x$ and the point $y$ defined $^{1}$ by

$$
y=-a x_{u}-b x_{v}+x_{u v}
$$

and the line $l_{2}$ joins the points $\rho, \sigma$ defined by placing

$$
\rho=x_{u}-b x, \quad \sigma=x_{v}-a x,
$$

where $a, b$ are functions of $u, v$. As $u, v$ vary, the lines $l_{1}, l_{2}$ generate two rectilinear congruences $\Gamma_{1}, \Gamma_{2}$ which are reciprocal with respect to the surface.

The curves corresponding to the developables of the congruence $\Gamma_{1}$ are called the $\Gamma_{1}$-curves of the congruence, and those corresponding to the developables of the congruence $\Gamma_{2}$ the $\Gamma_{2}$-curves of the congruence. The differential equation of the $\Gamma_{1}$-curves is

$$
(F-2 a \beta+\beta \psi) d u^{2}-\left(b_{v}-a_{u}\right) d u d v-(G-2 b \gamma+\gamma \phi) d v^{2}=0,
$$

where $F, G$ are defined by the formulas

$$
F=p-b_{u}+b \theta_{u}-b^{2}+a \beta, \quad G=q-a_{v}+a \theta_{v}-a^{2}+b \gamma,
$$

and $\phi, \psi$ by

$$
\phi=\left(\log \beta \gamma^{2}\right)_{u}, \quad \psi=\left(\log \beta^{2} \gamma\right)_{v} .
$$

If $k_{1}, k_{2}$ are the roots of the equation

$$
k^{2}+(A+B) k+A B-(F-2 a \beta+\beta \psi)(G-2 b \gamma+\gamma \phi)=0,
$$

where the functions $A, B$ are defined by

$$
A=-a_{u}-a b+\beta \gamma+\theta_{u v}, \quad B=-b_{v}-a b+\beta \gamma+\theta_{u v},
$$

the corresponding points

$$
y+k_{i} x, \quad i=1,2,
$$

are the focal points of the line $l_{1}$. Furthermore, the differential equation of the $\Gamma_{2}$-curves is

$$
F d u^{2}-\left(b_{v}-a_{u}\right) d u d v-G d v^{2}=0 .
$$

If $\tau_{1}, \tau_{2}$ are the roots of the equation

$$
F+\left(b_{v}-a_{u}\right) \tau-G \tau^{2}=0
$$

the corresponding points

$$
\rho+\tau_{i} \sigma, \quad i=1,2,
$$

${ }^{1}$ In this section we employ the notation used by E. P. Lane in Chapter III of his book Projective Differential Geometry of Curves and Surfaces, Chicago, 1932. 
are the focal points of the line $l_{2}$. It will be assumed that the coefficients of $d u^{2}$ and $d v^{2}$ in equations (5), (7) are all nonzero. In this case the $\Gamma_{1}$-curves and the $\Gamma_{2}$-curves of two reciprocal congruences form conjugate nets if, and only if, $b_{v}-a_{u}=0$.

3. Osculating quadrics of ruled surfaces of the congruence $\Gamma_{1}$. Any point $z$, except the point $y$, on the line $l_{1}$ at the point $x$ is given by the equation

$$
z=x+\omega y,
$$

$\omega$ scalar.

As the point $x$ varies along the $u$-curve, the line $l_{1}$ generates a ruled surface $R_{1}^{(u)}$. Equation (9) is the parametric vector equation of this ruled surface, $u, \omega$ being the independent parameters. The asymptotic curves on $R_{1}^{(u)}$ consist of the lines $l_{1}$ and the integral curves of the differential equation

$$
L_{1} d u+2 M_{1} d \omega=0,
$$

where $L_{1}, M_{1}$ are determinants of the fourth order defined by

$$
L_{1}=\left(z_{u u}, z, z_{u}, z_{\omega}\right), \quad M_{1}=\left(z_{u \omega}, z, z_{u}, z_{\omega}\right) .
$$

Differentiating equation (9) and using equations (2), (3) to calculate the values of $L_{1}, M_{1}$, we find that equation (10) can be written in the form

$$
\frac{d \omega}{d u}=-\frac{\beta+C \omega+D \omega^{2}}{2(F-2 a \beta+\beta \psi)},
$$

where we have placed

$$
\begin{aligned}
C= & F_{u}-2(a \beta)_{u}+(\beta \psi)_{u}+2 \beta A, \\
D= & \beta A^{2}-a(F-2 a \beta+\beta \psi)^{2}+A(F-2 a \beta+\beta \psi)_{u} \\
& -(F-2 a \beta+\beta \psi)\left[p_{v}+\beta q-a p+A\left(b-\theta_{u}\right)+A_{u}\right] .
\end{aligned}
$$

Any point $X$, except the point $z$, on the tangent at the point $z$ of the curved asymptotic on $R_{1}^{(u)}$ is defined by placing

$$
X=\lambda z+d z / d u,
$$

$\lambda$ scalar.

If we use the tetrahedron $x, \rho, \sigma, y$ as a local tetrahedron of reference with a unit point chosen so that a point

$$
x_{1} x+x_{2} \rho+x_{3} \sigma+x_{4} y
$$

has local coordinates proportional to $x_{1}, \cdots, x_{4}$, we find that the local coordinates of the point $X$ are given by 


$$
\begin{aligned}
& x_{1}=b+\lambda+\left[a(F-2 a \beta+\beta \psi)+b A+p_{v}+\beta q-a p\right] \omega, \\
& x_{2}=1+A \omega \\
& x_{3}=(F-2 a \beta+\beta \psi) \omega, \\
& x_{4}=\omega \lambda-\left(b-\theta_{u}\right) \omega-\frac{\beta+C \omega+D \omega^{2}}{2(F-2 a \beta+\beta \psi)} .
\end{aligned}
$$

Homogeneous elimination of $\omega, \lambda$ from these equations gives the algebraic equation of the quadric $Q_{1}^{(u)}$, referred to the tetrahedron $x, \rho, \sigma, y$, namely

$$
\begin{aligned}
\beta(F & -2 a \beta+\beta \psi) x_{2}^{2}+H x_{3}^{2}+2(F-2 a \beta+\beta \psi)^{2} x_{2} x_{4} \\
& -2 A(F-2 a \beta+\beta \psi) x_{3} x_{4} \\
& -2(F-2 a \beta+\beta \psi) x_{1} x_{3}+2 P x_{2} x_{3}=0
\end{aligned}
$$

where the coefficients $H, P$ are defined by

$$
\begin{aligned}
H & =a(F-2 a \beta+\beta \psi)-3 A\left(b-\theta_{u}\right)-A_{u}+p_{v}+\beta q-a p, \\
P & =\left(2 b-\theta_{u}\right)(F-2 a \beta+\beta \psi)+\frac{1}{2}(F-2 a \beta+\beta \psi)_{u} .
\end{aligned}
$$

The equation of the quadric $Q_{1}^{(0)}$ can be written by interchanging $u$ and $v$ and making the appropriate symmetrical interchanges of the other symbols. The result is

$$
\begin{aligned}
K x_{2}^{2} & +\gamma(G-2 b \gamma+\gamma \phi) x_{3}^{2}+2(G-2 b \gamma+\gamma \phi)^{2} x_{3} x_{4} \\
& -2 B(G-2 b \gamma+\gamma \phi) x_{2} x_{4} \\
& -2(G-2 b \gamma+\gamma \phi) x_{1} x_{2}+2 Q x_{2} x_{3}=0
\end{aligned}
$$

where the coefficients $K, Q$ are given by

$$
\begin{aligned}
K & =b(G-2 b \gamma+\gamma \phi)-3 B\left(a-\theta_{v}\right)-B_{v}+q_{v}+\gamma p-b q, \\
Q & =\left(2 a-\theta_{v}\right)(G-2 b \gamma+\gamma \phi)+\frac{1}{2}(G-2 b \gamma+\gamma \phi)_{v} .
\end{aligned}
$$

Some properties of the quadrics $Q_{1}^{(u)}, Q_{1}^{(v)}$ will now be deduced. In the first place, the tangent plane, $x_{4}=0$, intersects each of the quadrics in a conic. The conic of intersection of the tangent plane and the quadric $Q_{1}^{(u)}$ touches the $u$-tangent at the point $x$ and intersects the $v$-tangent in the point whose local coordinates are

$$
\left(\frac{1}{2} H, 0, F-2 a \beta+\beta \psi, 0\right) .
$$

Similarly, the quadric $Q_{1}^{(v)}$ is intersected by the tangent plane in a conic which is tangent to the $v$-tangent at the point $x$ and intersects the $u$-tangent in the point 


$$
\left(\frac{1}{2} K, G-2 b \gamma+\gamma \phi, 0,0\right) .
$$

The face $x_{3}=0$ of the tetrahedron of reference intersects the quadric $Q_{1}^{(u)}$ in the line $l_{1}$ and in the line whose equations are

$$
\beta x_{2}+2(F-2 a \beta+\beta \psi) x_{4}=0, \quad x_{3}=0 .
$$

Moreover, the face $x_{2}=0$ cuts the quadric $Q_{1}^{(u)}$ in the line $l_{1}$ and in the line which joins the point (18) to the point on the line $l_{1}$ with local coordinates

$$
(-A, 0,0,1) \text {. }
$$

Similarly, the face $x_{2}=0$ cuts the quadric $Q_{1}^{(v)}$ in the line $l_{1}$ and in the line

$$
\gamma x_{3}+2(G-2 b \gamma+\gamma \phi) x_{4}=0, \quad x_{2}=0 .
$$

The face $x_{3}=0$ cuts the quadric $Q_{1}^{(v)}$ in the line $l_{1}$ and in the line which passes through the point (19) and meets the line $l_{1}$ in the point

$$
(-B, 0,0,1) \text {. }
$$

The points (21), (23) are found to coincide if, and only if, $a_{u}=b_{v}$. Thus we reach the following conclusion:

The $\Gamma_{1}$-curves and the $\Gamma_{2}$-curves of two reciprocal rectilinear congru. ences form conjugate nets on the surface if, and only if, the points (21), (23) coincide.

It is well known that two nonsingular quadric surfaces having one, and only one, generator in common intersect elsewhere in a twisted cubic. Elimination of $x_{1}$ between equations (14), (16) gives the cubic cone projecting the curve of intersection of the two quadrics from the point $x$. This cone has the line $l_{1}$ for a double line, the equations of the nodal tangent planes along the line $l_{1}$ being given by

$$
(F-2 a \beta+\beta \psi) x_{2}^{2}-\left(b_{v}-a_{u}\right) x_{2} x_{3}-(G-2 b \gamma+\gamma \phi) x_{3}^{2}=0 .
$$

A glance at equation (5) suffices to substantiate the following statement:

The nodal tangent planes along the double line $l_{1}$ of the cone projecting the curve of intersection of the quadrics $Q_{1}^{(u)}, Q_{1}^{(v)}$ from the point $x$ are the planes which intersect the tangent plane of the surface at the point $x$ in the tangents of the $\Gamma_{1}$-curves.

Eliminating $x_{2}$ from equations (14), (16), we obtain the equation of the cone which projects the curve of intersection of the quadrics 
$Q_{1}^{(u)}, Q_{1}^{(v)}$ from the vertex $\rho$ of the tetrahedron of reference. This projecting cone is found to be a composite quartic cone, one component being the face $x_{3}=0$ of the tetrahedron of reference. The other component is a cubic cone which is intersected by the face $x_{2}=0$ in a plane cubic curve. Placing $x_{3}=0$ in the equation of this curve, we find the intersections of the curve with the line $l_{1}$. It is now easy to verify the conclusion :

The quadrics $Q_{1}^{(u)}, Q_{1}^{(v)}$ intersect in the line $l_{1}$ and in a twisted cubic which crosses the line $l_{1}$ in its two focal points.

4. Osculating quadrics of ruled surfaces of the congruence $\Gamma_{2}$. The equations of the quadrics $Q_{2}^{(u)}, Q_{2}^{(v)}$ can be found without difficulty by applying the method of the preceding section. The details of the calculation need not be reproduced here, but the required equation of the quadric $Q_{2}^{(u)}$, referred to the tetrahedron $x, \rho, \sigma, y$, is found to be

$$
\beta x_{1}^{2}-2 F x_{1} x_{3}+2 F^{2} x_{2} x_{4}+2\left(a b-a_{u}\right) F x_{3} x_{4}+2 S x_{1} x_{4}+L x_{4}^{2}=0,
$$

where the functions $S, L$ are defined by

$$
\begin{aligned}
& S=\frac{1}{2} F_{u}-F \theta_{u}+2 b F-\beta\left(a b-a_{u}\right) \\
& L=\beta F G+F F_{v}-F\left(\theta_{u}-2 b\right)\left(b_{v}-a_{u}\right)+F\left(b_{v}-a_{u}\right)_{u} \\
& \quad-2 S\left(a b-a_{u}\right)-\beta\left(a b-a_{u}\right)^{2} .
\end{aligned}
$$

The equation of the quadric $Q_{2}^{(v)}$ is

$$
\gamma x_{1}^{2}-2 G x_{1} x_{2}+2 G^{2} x_{3} x_{4}+2\left(a b-b_{v}\right) G x_{2} x_{4}+2 T x_{1} x_{4}+M \stackrel{2}{x_{4}}=0
$$

where

$$
\begin{aligned}
T= & \frac{1}{2} G_{v}-G \theta_{v}+2 a G-\gamma\left(a b-b_{v}\right) \\
M= & \gamma F G+G G_{u}-G\left(\theta_{v}-2 a\right)\left(a_{u}-b_{v}\right)+G\left(a_{u}-b_{v}\right)_{v} \\
& -2 T\left(a b-b_{v}\right)-\gamma\left(a b-b_{v}\right)^{2} .
\end{aligned}
$$

The quadric $Q_{2}^{(u)}$ is intersected by the tangent plane in the line $l_{2}$ and also in the line

$$
\beta x_{1}-2 F x_{3}=0, \quad x_{4}=0 .
$$

The face $x_{3}=0$ cuts the quadric $Q_{2}^{(u)}$ in a conic which is tangent to the $u$-tangent at the point $\rho$ and which intersects the edge $x_{1}=x_{3}=0$ in the point with local coordinates

$$
\left(0, L, 0,-2 F^{2}\right)
$$


The face $x_{1}=0$ cuts the quadric $Q_{2}^{(u)}$ in the line $l_{2}$ and in the line which joins the point

$$
\left(0, a_{u}-a b, F, 0\right)
$$

on the line $l_{2}$ to the point (30).

Similarly, the tangent plane intersects the quadric $Q_{2}^{(v)}$ in the line $l_{2}$ and in the line

$$
\gamma x_{1}-2 G x_{2}=0, \quad x_{4}=0 .
$$

The plane $x_{2}=0$ cuts this quadric in a conic which is tangent to the $v$-tangent at the point $\sigma$ and which intersects the edge $x_{1}=x_{2}=0$ in the point

$$
\left(0,0, M,-2 G^{2}\right) \text {. }
$$

The face $x_{1}=0$ intersects the quadric $Q_{2}^{(v)}$ in the line $l_{2}$ and in the line which joins the point

$$
\left(0, G, b_{v}-a b, 0\right)
$$

on the line $l_{2}$ to the point (33). The following conclusion is immediate.

If the points (31), (34) coincide respectively with the points $\sigma, \rho$, the $\Gamma_{1}$-curves and the $\Gamma_{2}$-curves form conjugate nets.

Elimination of $x_{2}$ from equations (25), (27) yields the equation of the cubic cone projecting from the point $\rho$ the curve of intersection of the quadrics $Q_{2}^{(u)}, Q_{2}^{(v)}$. The line $l_{2}$ is a double line of this cone, the nodal tangent planes along the line $l_{2}$ being given by

$$
x_{1}^{2}-\left(b_{v}-a_{u}\right) x_{1} x_{4}-\left[F G+\left(a b-b_{v}\right)\left(a b-a_{u}\right)\right] x_{4}^{2}=0 .
$$

It is now easy to verify the conclusion:

The two nodal tangent planes along the double line $l_{2}$ of the cone projecting the curve of intersection of the quadrics $Q_{2}^{(u)}, Q_{2}^{(v)}$ from the point $\rho$ intersect the line $l_{1}$ in two points which separate the points $x, y$ harmonically if, and only if, the $\Gamma_{1}$-curves and the $\Gamma_{2}$-curves form conjugate nets.

Finally, simple calculations suffice to demonstrate the following theorem:

The quadrics $Q_{2}^{(u)}, Q_{2}^{(v)}$ intersect in the line $l_{2}$ and in a twisted cubic which cuts the line $l_{2}$ in its two focal points.

5. A special case. The theory of the preceding sections will now be specialized by considering a particular covariant pair of reciprocal 
lines associated with the point $x$ of the surface. It is known that the line $l_{1}$ is the projective normal and the line $l_{2}$.is the reciprocal projective normal in case $a=b=0$ in equations (3), (4). Placing $a=b=0$ in equations (14), (16), one easily shows that the equations of the two quadrics $Q_{1}^{(u)}, Q_{1}^{(v)}$, which we shall call the projective normal quadrics, are respectively

$$
\begin{aligned}
\beta \pi x_{2}^{2} & +\left(p_{v}+\beta q+3 \theta_{u} k-k_{u}\right) x_{3}^{2}+2 \pi^{2} x_{2} x_{4}+\left(\pi_{u}-2 \pi \theta_{u}\right) x_{2} x_{3} \\
& -2 \pi k x_{3} x_{4}-2 \pi x_{1} x_{3}=0, \\
\gamma \chi x_{3}^{2} & +\left(q_{u}+\gamma p+3 \theta_{v} k-k_{v}\right) x_{2}^{2}+2 \chi^{2} x_{3} x_{4}+\left(\chi_{v}-2 \chi \theta_{v}\right) x_{2} x_{3} \\
& -2 \chi k x_{2} x_{4}-2 \chi x_{1} x_{2}=0,
\end{aligned}
$$

where $\pi, \chi, k$ are defined by the formulas

$$
\pi=p+\beta \psi, \quad \chi=q+\gamma \phi, \quad k=\beta \gamma+\theta_{u v} .
$$

Moreover, by placing $a=b=0$ in equations (25), (27), we obtain the two reciprocal projective normal quadrics $Q_{2}^{(u)}, Q_{2}^{(v)}$, whose equations are respectively

$$
\begin{aligned}
& \beta x_{1}^{2}+p\left(p_{v}+\beta q\right) x_{4}^{2}+\left(p_{u}-2 p \theta_{u}\right) x_{1} x_{4}-2 p x_{1} x_{3}+2 p^{2} x_{2} x_{4}=0 \\
& \gamma x_{1}^{2}+q\left(q_{u}+\gamma p\right) x_{4}^{2}+\left(q_{v}-2 q \theta_{v}\right) x_{1} x_{4}-2 q x_{1} x_{2}+2 q^{2} x_{3} x_{4}=0 .
\end{aligned}
$$

Southwestern College 